\section{Escala de Influência dos Três Fatores (TIS) aplicada a estudantes universitários: estudo de validação e aplicação}

\author{
Tripartite Influence Scale (TIS) applied to university \\ students: validation study and application
}

\author{
Escala de Influencia de los Tres Factores (TIS) \\ aplicada a estudiantes universitarios: \\ estudio de validación y aplicación
}

QUESTÕES METODOLÓGICAS

METHODOLOGICAL ISSUES

\section{Resumo}

A Escala de Influência dos Três Fatores (TIS) é utilizada para avaliar a influência de aspectos sociais e familiares na aparência corporal dos indivíduos. Contudo, poucos indicios de validade de construto da TIS são apresentados. O objetivo do estudo foi estimar os indicadores psicométricos da TIS e identificar o grau de influência da mídia, pais e amigos na aparência corporal de universitários considerando diferentes características. Universitários de ambos os sexos participaram. Os indicadores psicométricos da TIS foram avaliados para cada sexo utilizando análise fatorial confirmatória. Os escores médios de cada fator da TIS foram calculados. As prevalências do grau de influência foram apresentadas. Um modelo de regressão múltiplo multivariado foi construído para verificar a relação de características de interesse com os escores dos fatores da TIS. Participaram 791 universitários (63,2\% mulheres) de 18 a 40 anos. A TIS não apresentou bom ajustamento às amostras e foi refinada. Após refinamento, observou-se adequada validade e confiabilidade para mulheres e homens. Verificaram-se diferenças significativas entre os escores médios de mulheres e homens, e, nas prevalências, a maioria dos estudantes esteve na categoria baixa. A atividade laboral, o consumo de suplementos alimentares para alterar o corpo, a idade, o indice de massa corporal, a autoavaliação da alimentação e o nível de atividade física se relacionaram significativamente com os fatores da TIS. Em geral, as mulheres foram mais influenciadas pela mídia e pelos amigos do que os homens. As características amostrais identificadas como relacionadas aos fatores da TIS podem ser relevantes para inclusão em protocolos clínicos e de pesquisa.

Imagem Corporal; Estudantes; Estudos de Validação
Wanderson Roberto da Silva 1

João Marôco 2

Juliana Alvares Duarte Bonini Campos 1

\author{
Correspondência \\ J. A. D. B. Campos \\ Universidade Estadual Paulista Júlio de Mesquita Filho. \\ Rod. Araraquara-Jaú, Araraquara, SP 14800-903, Brasil. \\ jucampos@fcfar.unesp.br \\ 1 Universidade Estadual Paulista Júlio de Mesquita Filho, \\ Araraquara, Brasil. \\ 2 Instituto Universitário de Ciências Psicológicas, Sociais e da \\ Vida, Lisboa, Portugal.
}




\section{Introdução}

A busca para entender a percepção dos indivíduos quanto ao próprio corpo é comum entre os estudiosos de imagem corporal 1. A maioria das pesquisas nessa área investiga a relação que as pessoas têm com o corpo, mas outros estudos que buscam identificar os motivos que podem influenciar no desejo de modificar a aparência do corpo têm ganhado espaço nos últimos anos. Existem inúmeros motivos que levam as pessoas a modificarem o corpo, e, entre esses, podem ser destacados a influência dos meios de comunicação de massa e o convívio com amigos e familiares 2,3. O modelo de influência tripartido 4,5 é comumente utilizado para avaliar as pressões midiáticas e interpessoais que podem influenciar na construção dos ideais de aparência dos indivíduos e favorecer a insatisfação corporal devido à internalização e à adoção de padrões dificilmente alcançáveis. Esse modelo tem sido avaliado em diferentes amostras incluindo universitários do sexo feminino ${ }^{6}$ e masculino ${ }^{7}$. Além disso, a literatura 1,8,9 sugere que, quando da avaliação de aspectos relacionadas à imagem corporal, é interessante realizar a investigação considerando as peculiaridades existentes entre mulheres e homens, ou seja, as avaliações devem ser realizadas separadas.

Thompson et al. 5 e van den Berg et al. ${ }^{3}$ destacam que identificar a influência da mídia, família e amigos na aparência corporal é importante para compreender a relevância desses contextos na internalização e comparação corporal das pessoas e, assim, conseguir intervir na insatisfação corporal. Essa intervenção poderá auxiliar na redução dos casos de transtornos de imagem e alimentação que causam prejuízos à saúde física e mental dos indivíduos 1,10,11. No Brasil, as pessoas valorizam fortemente a aparência corporal, e isso é um dos principais fatores para a adoção de comportamentos de risco como, por exemplo, a restrição severa do consumo alimentar 12. Nesse contexto, investigar a aparência corporal considerando, por exemplo, as influências interpessoais 13 torna-se relevante para a saúde da população brasileira, uma vez que, a partir da identificação dessas, pode-se desenvolver medidas educativas e/ou preventivas para minimizar/evitar a instalação de comportamentos disfuncionais em relação à alimentação e à imagem corporal. Uma das formas de identificar a influência de aspectos socioculturais na aparência corporal é por meio da utilização de instrumentos psicométricos. Entre esses, podem-se destacar a Perceived Sociocultural Pressures-Family subscale (PSP-F) 14, a Family, Peers and Media Influence Scale-Family subscale (FPM-F) 15, o Sociocultural Attitudes Towards Appearance Questionnaire 4 (SATAQ-4) 16 e a Tripartite Influence Scale (TIS) 17.

A TIS foi construída por Shroff \& Thompson (2003, apud, Keery et al. 17) a partir de outras escalas da mesma temática para avaliar o quanto a mídia e o convívio com pais e amigos podem influenciar na aparência corporal dos indivíduos. A escala foi apresentada por Keery et al. 17 e testada, inicialmente, em uma amostra de adolescentes americanas. Posteriormente, outros estudos utilizaram a TIS 6,18,19,20, contudo, em nenhuma das pesquisas, foi avaliada a validade de construto do instrumento utilizando estratégia confirmatória. Em contexto brasileiro, a TIS foi traduzida para o português em estudo de adaptação transcultural em que foi também verificada sua validade de conteúdo, mas não foi confirmada sua validade fatorial 18 . Na sequência, outro estudo 21 explorou a estrutura fatorial da escala e encontrou a presença dos três fatores conforme a proposta original. Entretanto, esse estudo não apresentou estimativas de validade de construto do modelo original da TIS para o contexto brasileiro, sendo essa uma lacuna.

Cabe relatar ainda, em relação às estimativas de validade de construto da TIS, que a estratégia confirmatória, além de fornecer informações relevantes sobre o modelo teórico proposto, também pode apresentar evidências para sustentar a qualidade dos resultados obtidos com a TIS, fortalecendo a utilização do instrumento em diferentes contextos. Nesse sentido, vale destacar que avaliar a validade e a confiabilidade de um instrumento de medida vai além de expor a validade de conteúdo ou estimativas de consistência interna e teste-reteste. Além disso, deve-se destacar que esses indicadores não são propriedades do instrumento de medida, mas apenas desse aplicado a determinado contexto ou amostra 22. Portanto, identificar se o instrumento apresenta validade de construto (fatorial, convergente, discriminante) e confiabilidade para cada contexto torna-se indispensável. Após identificar se os dados podem ser considerados válidos, abre-se espaço para a utilização do instrumento.

Ainda, no que se refere à influência da mídia e do convívio familiar e social na aparência corporal, vale mencionar a relevância que características demográficas (p.ex.: sexo) e antropométricas (p.ex.: 
índice de massa corporal - IMC) podem exercer nesses contextos. Estudos anteriores 23,24,25,26 têm apontado que os indivíduos classificados em sobrepeso/obesidade e as mulheres são mais vulneráveis às pressões sociais e culturais voltadas aos padrões estéticos, ou seja, são grupos que necessitam de maior atenção. Além disso, outras características como, por exemplo, atividade laboral, classe econômica e nível de atividade física podem impactar na aparência corporal, e, portanto, investigar tais variáveis pode ser interessante 27,28 .

Frente ao exposto anteriormente, realizou-se este estudo com os objetivos de estimar os indicadores psicométricos da TIS para uma amostra de estudantes universitários brasileiros e identificar o grau de influência da mídia, pais e amigos na aparência corporal dos estudantes considerando características demográficas e antropométricas.

\section{Método}

\section{Desenho de estudo e tamanho amostral}

O presente estudo é observacional do tipo transversal com amostragem não probabilística delineada por conveniência. O tamanho amostral mínimo foi calculado utilizando, pelo menos, cinco sujeitos por item da TIS 29. Considerando que a escala é composta originalmente por 43 itens, o tamanho amostral mínimo foi estimado em 215 participantes. Essa estimativa foi respeitada para cada sexo, pois todas as análises foram realizadas separadamente para mulheres e homens.

\section{Participantes}

Foram convidados a participar da pesquisa estudantes universitários, de ambos os sexos, matriculados nos cursos de graduação da Faculdade de Ciências Farmacêuticas e da Faculdade de Ciências e Letras, Universidade Estadual Paulista (UNESP/Araraquara). Os critérios de inclusão adotados foram ter idade entre 18 e 40 anos, não estar em período de gestação ou lactação, não possuir deficiência visual grave e não estar em tratamento para nenhum tipo de transtorno alimentar durante a coleta de dados.

\section{Caracterização da amostra de estudo}

Informações referentes a sexo, idade, curso, ano e turno frequentado, presença de atividade laboral concomitante aos estudos, moradia (sozinho, família ou amigos/colegas), autoavaliação da alimentação (ruim, regular, normal, boa e excelente), consumo de suplementos alimentares para alterar o corpo e realização e nível de atividade física foram levantadas. O peso corporal e a altura também foram informados pelos estudantes para cálculo do IMC e posterior classificação do estado nutricional antropométrico 30,31. O Critério Brasil (Associação Brasileira de Empresas de Pesquisa. http:// www.abep.org/, acessado em 04/Abr/2018) foi utilizado para identificar a classe econômica de cada participante. O nível de atividade física dos estudantes foi estimado utilizando a versão reduzida do International Physical Activity Questionnaire (IPAQ).

\section{Instrumento de medida}

Para avaliar a influência da mídia, pais e amigos na aparência corporal, foi utilizada a TIS. O instrumento foi proposto para a população jovem americana, na língua inglesa, com o objetivo de avaliar a influência que os meios de comunicação de massa e o convívio com membros da família e amigos podem exercer na aparência corporal dos indivíduos. A escala original apresenta 43 itens distribuídos em três fatores (Mídia: itens 1-10; Pais: itens 11-30; Amigos: itens 31-43). A opção de respostas aos itens é apresentada em escala do tipo Likert de cinco pontos. A versão em português da TIS foi desenvolvida por Conti et al. 18 e denominada Escala de Influência dos Três Fatores (EITF). Contudo, a versão final apresentada em português para o contexto brasileiro possui 39 itens, e a justificativa para eliminação de quatro itens (itens: 27, 30, 39 e 43) foi baseada na redundância teórica deles a partir 
de análise de conteúdo. Sabendo-se que os indicadores psicométricos de um instrumento de medida estão diretamente relacionados às características da amostra de estudo e que esses não foram avaliados no estudo de Conti et al. 18 , entende-se que não existe evidência suficiente (relacionada à validade de construto) que sustente a adequação da estrutura composta por 39 itens. Assim, neste estudo, foi utilizada a versão traduzida em português 18 , porém, o modelo testado foi o original, com todos os itens. Além disso, nós utilizamos a sigla original (TIS) como referência ao instrumento.

\section{Validação de face da TIS}

Inicialmente, foi realizado processo de validação de face da TIS com o objetivo de verificar as equivalências conceitual e cultural da versão completa em português do instrumento apresentada por Conti et al. 18 com a versão original em inglês 32,33 . Para tanto, três especialistas em imagem corporal e psicometria avaliaram os itens, a escala de resposta e a composição dos fatores da TIS, considerando o contexto universitário ao qual seria aplicada. Os especialistas consideraram os itens e a composição dos fatores adequados, contudo, destacaram que as opções de resposta não eram claras e sugeriram algumas alterações. Conti et al. 18 relatam que as opções de resposta para todos os itens da TIS são $1=$ sempre, 2 = quase sempre, 3 = frequentemente, 4 = algumas vezes e $5=$ nunca. Entretanto, segundo os especialistas, somente dez itens (do 1 ao 10) estão devidamente direcionados a esse tipo de escala de resposta de "frequência", sendo os demais mais voltados a uma escala de resposta de "intensidade". Ainda, os especialistas destacaram que o valor numérico atribuído a cada opção de resposta não estava acompanhando, de forma direta, o grau de magnitude, por exemplo, o número 1 era atribuído a "sempre" enquanto o número 5 a "nunca".

Assim, foram sugeridas duas modificações no instrumento, a primeira foi referente à separação dos itens a partir de duas escalas de resposta do tipo Likert de cinco pontos, sendo, do item 1 ao 10 , utilizada escala de "frequência" ( 1 = nunca, 2 = algumas vezes, $3=$ frequentemente, 4 = quase sempre, 5 = sempre) e, do item 11 ao 43 , a escala de "intensidade" ( 1 = nada, 2 = pouco, 3 = moderadamente, $4=$ muito, 5 = muitíssimo). A segunda modificação tratou da inversão dos valores numéricos atribuídos às respostas aos itens buscando manter o sentido direto entre a pontuação e o grau de magnitude.

Depois de realizadas as modificações sugeridas, a versão em português completa da TIS foi prétestada em estudo piloto realizado com 30 universitários de ambos os sexos. Nenhum participante relatou dificuldade para o preenchimento dos itens ou opções de resposta e, portanto, a versão foi considerada adequada. É importante destacar que, nessa versão, quanto maior o escore geral obtido, maior a influência na aparência corporal do indivíduo.

\section{Procedimentos e aspectos éticos}

A versão em português completa da TIS e as perguntas utilizadas para caracterização dos participantes foram apresentadas aos estudantes em sala de aula em horário habitual das aulas teóricas. Todos os estudantes presentes durante a coleta de dados foram convidados, voluntariamente, a participar do estudo. O convite foi realizado verbalmente pelos pesquisadores do estudo que informaram os objetivos da pesquisa. Os estudantes que concordaram em participar assinaram o Termo de Consentimento Livre e Esclarecido e preencheram os dados. Cabe mencionar que todas as coletas foram realizadas com a presença de um professor que concordou previamente em fornecer 15 minutos de sua aula para a participação dos estudantes na pesquisa.

Todos os aspectos éticos para a realização do estudo foram respeitados, e esse foi aprovado pelo Comitê de Ética em Pesquisa em Seres Humanos da Faculdade de Ciências Farmacêuticas da UNESP (C.A.A.E. $46774015.5 \cdot 0000.5426)$.

\section{Análise estatística}

A análise descritiva de todos os itens da TIS foi realizada a partir da média, mediana, desvio padrão, assimetria e curtose. Valores absolutos de assimetria e curtose maiores que três e sete, respectivamente, foram considerados indicativos de violação da normalidade 34 . 
A validade fatorial da TIS foi avaliada por meio de análise fatorial confirmatória (AFC) utilizando o método de estimação weighed least squares mean and variance adjusted. O modelo original composto por 43 itens e três fatores (modelo oblíquo) foi testado. Todas as análises foram realizadas separadamente para mulheres e homens.

$\mathrm{O}$ ajustamento do modelo às amostras foi verificado considerando os índices: razão de qui-quadrado pelos graus de liberdade $\left(\chi^{2} / \mathrm{gl}\right)$, root mean square error of approximation (RMSEA) com intervalo de 90\% de confiança (IC90\%), Tucker-Lewis index (TLI) e comparative fit index (CFI) 35. Para avaliar a qualidade do ajustamento do modelo fatorial nas amostras, os valores de $\chi^{2} / \mathrm{gl} \leq 5,0$, RMSEA $\leq 0,10$, TLI $\geq 0,90$ e CFI $\geq 0,90$ foram considerados indicadores de um ajustamento aceitável 34. Também foram calculados e analisados os pesos fatoriais $(\lambda)$ de cada item da TIS, sendo aqueles inferiores a 0,40 eliminados. Quando o modelo não apresentou bom ajustamento, os índices de modificação calculados a partir dos multiplicadores de Lagrange foram analisados. Essas análises foram conduzidas no programa Mplus, versão 7.2 (https://www.statmodel.com/).

As validades convergente e discriminante também foram analisadas. Para tanto, foram calculados a variância extraída média (VEM) e o coeficiente de determinação ( $\left.\mathrm{r}^{2}\right)$ de cada fator da TIS. Em segui$\mathrm{da}$, as estimativas foram avaliadas, e os valores de $\mathrm{VEM} \geq 0,50$ foram considerados indicativos de adequada validade convergente, enquanto os valores de $\mathrm{r}^{2} \leq$ VEM de cada par de fatores correlacionados indicaram adequada validade discriminante 34,36 .

A confiabilidade de cada fator da TIS foi analisada por meio das estimativas obtidas a partir do coeficiente alfa ordinal $(\alpha)$ e da confiabilidade composta (CC). Para cálculo do $\alpha$, os pacotes semTools 37 e lavaan 38 foram utilizados no programa R (http://www.r-project.org). Valores de $\alpha$ e CC $\geq 0,70$ foram considerados indicativos de adequada confiabilidade 34,36 .

Após ajustamento do modelo da TIS aos dados, os escores finais para todos os fatores da escala foram estimados para cada sexo utilizando a média aritmética. Esses escores foram comparados segundo o sexo utilizando teste t de Student e $\alpha=5 \%$. Para a classificação dos indivíduos segundo o grau de influência da mídia, pais e amigos na aparência corporal, foram considerados os percentis 25, 50 e 75 das opções de resposta da TIS. A pontuação e classificação para cada fator da TIS foram: $1,0 \mathrm{H}$ 2,0 pontos $(\leq \mathrm{P} 25)=$ muito baixa; 2,0- 3,0 (P25- P50) = baixa; 3,0才 4,0 (P50- P75) = moderada; 4,0- 5,0 (> P75) = alta. Em seguida, a prevalência de estudantes classificados com muito baixa, baixa, moderada e alta influência da mídia, pais e amigos na aparência corporal para cada sexo foi calculada por ponto e por intervalo de $95 \%$ de confiança (IC95\%).

Para identificar a relação de características demográficas e antropométricas e os escores médios dos fatores da TIS, foi elaborado um modelo de regressão múltiplo multivariado. Esse modelo foi construído para verificar se a idade, a atividade laboral, o consumo de suplementos alimentares para alterar o corpo, a autoavaliação da alimentação, o IMC, o nível de atividade física e a classe econômica (variáveis independentes) poderiam se relacionar significativamente com o escore médio de cada fator da TIS (variáveis dependentes: mídia, pais e amigos). O modelo foi confeccionado para mulheres e homens, separadamente, no programa Mplus, versão 7.2, e utilizou-se a máxima verossimilhança como método de estimação. A avaliação do modelo foi realizada considerando as trajetórias hipoteticamente causais $(\beta)$, e aquelas não significativas $(\mathrm{p}<0,05)$ foram eliminadas 34 .

\section{Resultados}

Participaram do estudo 791 estudantes, sendo a maioria do sexo feminino (62,2\%). A média de idade foi de 21,1 (DP = 2,9) anos para as mulheres e de 21,4 (DP = 3,8) anos para os homens. Na Tabela 1, apresenta-se a caracterização da amostra de estudo. Observa-se, que a maioria dos estudantes frequentava o primeiro ano do curso, estudava no período noturno, residia com amigos ou colegas, relatou não trabalhar, nunca ter consumido suplementos alimentares para alterar o corpo, classificou a alimentação como normal, praticava atividade física, foi classificada segundo o nível de atividade física como alto, segundo o estado nutricional como eutrofia e segundo a classe econômica no nível B.

$\mathrm{Na}$ Tabela 2, encontra-se a análise descritiva das respostas dadas aos itens da TIS. Nota-se que apenas o item 34 para amostra feminina e o item 10 para a amostra masculina apresentaram valores discrepantes de curtose. 
Tabela 1

Caracterização da amostra de estudantes universitários separada por sexo.

\begin{tabular}{|c|c|c|}
\hline Característica & $\begin{array}{c}\text { Feminina } \\
\text { n (\%) }\end{array}$ & $\begin{array}{c}\text { Masculina } \\
\text { n (\%) }\end{array}$ \\
\hline \multicolumn{3}{|l|}{ Curso } \\
\hline Farmácia & $123(25,3)$ & $30(10,0)$ \\
\hline Administração & $79(16,2)$ & $82(27,4)$ \\
\hline Letras & $3(0,6)$ & $2(0,7)$ \\
\hline Pedagogia & $138(28,3)$ & $4(1,3)$ \\
\hline Economia & $58(11,9)$ & $106(35,5)$ \\
\hline Ciências Sociais & $42(8,6)$ & $56(18,7)$ \\
\hline Engenharia de Bioprocessos & $44(9,1)$ & $19(6,4)$ \\
\hline \multicolumn{3}{|l|}{ Ano do curso } \\
\hline 10 & $156(32,1)$ & $120(40,1)$ \\
\hline 20 & $106(21,7)$ & $66(22,1)$ \\
\hline 3으 & $67(13,7)$ & $51(17,1)$ \\
\hline 4 으 & $128(26,2)$ & $40(13,4)$ \\
\hline$>50$ & $31(6,3)$ & $22(7,3)$ \\
\hline \multicolumn{3}{|l|}{ Turno do curso } \\
\hline Integral & $149(30,3)$ & $58(19,4)$ \\
\hline Manhã/Tarde & $167(33,9)$ & $91(30,4)$ \\
\hline Noite & $176(35,8)$ & $150(50,2)$ \\
\hline \multicolumn{3}{|l|}{ Moradia } \\
\hline Sozinho & $60(12,2)$ & $57(19,1)$ \\
\hline Família & $208(42,4)$ & $106(35,6)$ \\
\hline Amigos/Colegas & $223(45,4)$ & $135(45,3)$ \\
\hline \multicolumn{3}{|l|}{ Atividade laboral } \\
\hline Não & $393(80,5)$ & $237(79,8)$ \\
\hline Sim & $95(19,5)$ & $60(20,2)$ \\
\hline \multicolumn{3}{|c|}{ Consumo de suplementos para alterar o corpo } \\
\hline Nunca & $376(78,0)$ & $184(62,5)$ \\
\hline Uma vez na vida & $41(8,5)$ & $37(12,6)$ \\
\hline Às vezes & $45(9,3)$ & $54(18,4)$ \\
\hline Com frequência & $20(4,2)$ & $19(6,5)$ \\
\hline \multicolumn{3}{|c|}{ Como você considera sua alimentação? } \\
\hline Ruim & $43(9,1)$ & $41(13,9)$ \\
\hline Regular & $153(32,3)$ & $68(23,1)$ \\
\hline Normal & $168(35,4)$ & $89(30,3)$ \\
\hline Boa & $99(20,9)$ & $85(28,9)$ \\
\hline Excelente & $11(2,3)$ & $11(3,8)$ \\
\hline \multicolumn{3}{|l|}{ Estado nutricional antropométrico } \\
\hline Desnutrição & $23(4,7)$ & $9(3,1)$ \\
\hline Eutrofia & $350(72,2)$ & $195(66,3)$ \\
\hline Sobrepeso & $83(17,1)$ & $65(22,1)$ \\
\hline Obesidade & $29(6,0)$ & $25(8,5)$ \\
\hline \multicolumn{3}{|l|}{ Prática de atividade física } \\
\hline Não & $207(42,5)$ & $88(29,4)$ \\
\hline Sim & $280(57,5)$ & $211(70,6)$ \\
\hline \multicolumn{3}{|l|}{ Nível de atividade física } \\
\hline Baixo & $50(11,0)$ & $22(7,7)$ \\
\hline Moderado & $158(34,8)$ & $72(25,3)$ \\
\hline Alto & $246(54,2)$ & $191(67,0)$ \\
\hline \multicolumn{3}{|c|}{ Classe econômica (renda média mensal/2016) } \\
\hline D e $E(R \$ 768,00)$ & $1(0,2)$ & $1(0,4)$ \\
\hline$C(R \$ 1.625,00 / R \$ 2.705,00)$ & $106(22,5)$ & $41(14,4)$ \\
\hline $\mathrm{B}(\mathrm{R} \$ 4.852,00 / \mathrm{R} \$ 9.254,00)$ & $238(50,4)$ & $154(54,2)$ \\
\hline$A(R \$ 20.888,00)$ & $127(26,9)$ & $88(31,0)$ \\
\hline
\end{tabular}


Tabela 2

Estatística descritiva e pesos fatoriais padronizados dos itens da Tripartite Influence Scale (TIS) separados por sexo.

\begin{tabular}{|c|c|c|c|c|c|c|c|c|c|c|c|c|c|c|}
\hline \multirow[t]{2}{*}{ Item } & \multicolumn{7}{|c|}{ Feminino $(n=492)$} & \multicolumn{7}{|c|}{ Masculino $(n=299)$} \\
\hline & Média & Mediana & DP & Assimetria & Curtose & $\lambda_{c}$ & $\lambda_{\mathrm{a}}$ & Média & Mediana & DP & Assimetria & Curtose & $\lambda_{c}$ & $\lambda_{a}$ \\
\hline 1 & 2,85 & 3,00 & 1,27 & 0,35 & $-0,98$ & 0,84 & 0,63 & 2,35 & 2,00 & 1,23 & 0,74 & $-0,35$ & 0,82 & 0,65 \\
\hline 2 & 3,13 & 3,00 & 1,25 & 0,11 & $-1,08$ & 0,85 & 0,66 & 2,61 & 2,00 & 1,25 & 0,51 & $-0,73$ & 0,85 & 0,69 \\
\hline 3 & 2,66 & 2,00 & 1,22 & 0,44 & $-0,71$ & 0,67 & 0,76 & 2,13 & 2,00 & 1,14 & 0,83 & $-0,08$ & 0,73 & 0,78 \\
\hline 4 & 2,28 & 2,00 & 1,38 & 0,84 & $-0,58$ & 0,63 & - & 1,68 & 1,00 & 1,07 & 1,66 & 2,04 & 0,68 & - \\
\hline 5 & 1,74 & 1,00 & 1,05 & 1,64 & 2,23 & 0,84 & 0,88 & 1,39 & 1,00 & 0,80 & 2,42 & 6,08 & 0,82 & 0,84 \\
\hline 6 & 2,22 & 2,00 & 1,14 & 0,87 & 0,06 & 0,87 & 0,90 & 1,81 & 2,00 & 1,00 & 1,23 & 1,09 & 0,78 & 0,81 \\
\hline 7 & 2,38 & 2,00 & 1,22 & 0,69 & $-0,46$ & 0,92 & 0,45 & 1,39 & 1,00 & 0,80 & 2,37 & 5,87 & 0,79 & 0,59 \\
\hline 8 & 1,76 & 1,00 & 1,04 & 1,54 & 1,89 & 0,87 & 0,90 & 1,44 & 1,00 & 0,83 & 2,15 & 4,61 & 0,82 & 0,85 \\
\hline 9 & 2,15 & 2,00 & 1,08 & 0,90 & 0,25 & 0,87 & 0,90 & 1,76 & 1,00 & 0,94 & 1,21 & 0,96 & 0,79 & 0,83 \\
\hline 10 & 2,24 & 2,00 & 1,20 & 0,83 & $-0,24$ & 0,93 & 0,44 & 1,31 & 1,00 & 0,76 & 2,80 & 7,87 * & 0,82 & 0,62 \\
\hline 11 & 2,59 & 3,00 & 1,26 & 0,27 & $-0,92$ & 0,69 & 0,70 & 2,21 & 2,00 & 1,13 & 0,56 & $-0,54$ & 0,65 & 0,65 \\
\hline 12 & 2,15 & 2,00 & 1,14 & 0,80 & $-0,18$ & 0,75 & 0,76 & 1,98 & 2,00 & 1,00 & 0,88 & 0,33 & 0,67 & 0,68 \\
\hline 13 & 2,07 & 2,00 & 1,17 & 0,85 & $-0,25$ & 0,78 & 0,79 & 2,02 & 2,00 & 1,14 & 0,95 & 0,01 & 0,77 & 0,78 \\
\hline 14 & 1,85 & 1,00 & 1,07 & 1,17 & 0,57 & 0,79 & 0,80 & 1,88 & 2,00 & 1,04 & 1,12 & 0,70 & 0,85 & 0,85 \\
\hline 15 & 1,55 & 1,00 & 0,95 & 2,02 & 3,86 & 0,51 & - & 1,52 & 1,00 & 0,88 & 1,86 & 3,01 & 0,59 & - \\
\hline 16 & 1,52 & 1,00 & 0,84 & 1,85 & 3,44 & 0,65 & 0,51 & 1,50 & 1,00 & 0,87 & 2,02 & 4,09 & 0,71 & 0,59 \\
\hline 17 & 1,97 & 2,00 & 0,99 & 0,96 & 0,65 & 0,58 & 0,66 & 1,98 & 2,00 & 0,97 & 0,88 & 0,37 & 0,65 & 0,71 \\
\hline 18 & 2,02 & 2,00 & 1,21 & 1,09 & 0,20 & 0,69 & 0,59 & 1,97 & 2,00 & 1,07 & 0,95 & 0,13 & 0,64 & 0,66 \\
\hline 19 & 2,13 & 2,00 & 1,16 & 0,83 & $-0,18$ & 0,73 & 0,69 & 2,06 & 2,00 & 1,05 & 0,74 & $-0,30$ & 0,73 & 0,64 \\
\hline 20 & 2,80 & 3,00 & 1,14 & 0,31 & $-0,60$ & 0,69 & 0,67 & 2,66 & 3,00 & 1,12 & 0,22 & $-0,71$ & 0,66 & 0,66 \\
\hline 21 & 1,51 & 1,00 & 1,00 & 2,20 & 4,17 & 0,62 & 0,62 & 1,49 & 1,00 & 0,89 & 2,12 & 4,43 & 0,67 & 0,58 \\
\hline 22 & 1,71 & 1,00 & 1,08 & 1,67 & 2,06 & 0,64 & 0,62 & 1,48 & 1,00 & 0,85 & 2,06 & 4,35 & 0,67 & 0,67 \\
\hline 23 & 1,94 & 2,00 & 1,04 & 1,30 & 1,40 & 0,60 & 0,64 & 1,71 & 1,00 & 0,95 & 1,46 & 1,75 & 0,67 & 0,66 \\
\hline 24 & 1,91 & 2,00 & 1,12 & 1,32 & 1,11 & 0,72 & 0,61 & 1,75 & 1,00 & 0,95 & 1,27 & 1,25 & 0,73 & 0,67 \\
\hline 25 & 1,96 & 2,00 & 1,04 & 1,26 & 1,36 & 0,73 & 0,73 & 1,87 & 2,00 & 0,95 & 1,09 & 0,86 & 0,74 & 0,74 \\
\hline 26 & 2,45 & 2,00 & 1,08 & 0,80 & 0,24 & 0,77 & 0,73 & 2,32 & 2,00 & 1,01 & 0,73 & 0,28 & 0,68 & 0,74 \\
\hline 27 & 2,69 & 2,00 & 1,14 & 0,53 & $-0,45$ & 0,49 & 0,76 & 2,57 & 2,00 & 1,13 & 0,60 & $-0,31$ & 0,44 & 0,67 \\
\hline 28 & 1,89 & 2,00 & 1,04 & 1,24 & 1,07 & 0,75 & 0,75 & 1,87 & 2,00 & 0,93 & 1,23 & 1,60 & 0,68 & 0,68 \\
\hline 29 & 2,06 & 2,00 & 1,04 & 0,94 & 0,43 & 0,69 & 0,69 & 2,03 & 2,00 & 1,01 & 0,82 & 0,17 & 0,75 & 0,75 \\
\hline 30 & 2,23 & 2,00 & 0,96 & 0,66 & 0,20 & 0,75 & 0,76 & 2,08 & 2,00 & 0,93 & 0,60 & $-0,09$ & 0,80 & 0,81 \\
\hline 31 & 2,50 & 2,00 & 1,16 & 0,58 & $-0,44$ & 0,68 & 0,68 & 2,17 & 2,00 & 1,11 & 0,87 & 0,18 & 0,61 & 0,62 \\
\hline 32 & 2,27 & 2,00 & 1,14 & 0,62 & $-0,40$ & 0,77 & 0,79 & 2,00 & 2,00 & 1,00 & 0,78 & $-0,01$ & 0,68 & 0,69 \\
\hline 33 & 2,85 & 3,00 & 1,10 & 0,19 & $-0,57$ & 0,84 & 0,88 & 2,60 & 3,00 & 1,07 & 0,17 & $-0,59$ & 0,81 & 0,87 \\
\hline 34 & 1,32 & 1,00 & 0,68 & 2,69 & 8,60 * & 0,49 & - & 1,44 & 1,00 & 0,82 & 2,33 & 5,87 & 0,64 & - \\
\hline 35 & 2,20 & 2,00 & 1,06 & 0,95 & 0,56 & 0,72 & 0,73 & 1,84 & 2,00 & 0,95 & 1,23 & 1,43 & 0,77 & 0,78 \\
\hline 36 & 1,94 & 2,00 & 1,02 & 1,08 & 0,69 & 0,68 & 0,70 & 1,60 & 1,00 & 0,87 & 1,60 & 2,47 & 0,76 & 0,77 \\
\hline 37 & 2,25 & 2,00 & 1,04 & 0,79 & 0,22 & 0,81 & 0,83 & 1,79 & 2,00 & 0,91 & 1,19 & 1,23 & 0,85 & 0,86 \\
\hline 38 & 2,36 & 2,00 & 1,09 & 0,69 & $-0,04$ & 0,84 & 0,85 & 1,94 & 2,00 & 0,99 & 1,14 & 1,12 & 0,82 & 0,84 \\
\hline 39 & 2,40 & 2,00 & 0,98 & 0,73 & 0,42 & 0,69 & 0,69 & 2,08 & 2,00 & 0,94 & 0,77 & 0,39 & 0,71 & 0,72 \\
\hline 40 & 2,24 & 2,00 & 1,00 & 0,72 & 0,21 & 0,77 & 0,78 & 1,89 & 2,00 & 0,92 & 1,20 & 1,64 & 0,79 & 0,80 \\
\hline 41 & 2,55 & 2,00 & 1,12 & 0,57 & $-0,31$ & 0,46 & - & 2,44 & 2,00 & 1,15 & 0,47 & $-0,53$ & 0,50 & - \\
\hline 42 & 2,92 & 3,00 & 1,11 & 0,16 & $-0,74$ & 0,84 & 0,70 & 2,65 & 3,00 & 1,16 & 0,15 & $-0,82$ & 0,84 & 0,68 \\
\hline 43 & 2,87 & 3,00 & 1,07 & 0,17 & $-0,57$ & 0,91 & 0,80 & 2,60 & 3,00 & 1,12 & 0,21 & $-0,70$ & 0,92 & 0,81 \\
\hline
\end{tabular}

$\lambda_{a}$ : peso fatorial dos itens do modelo ajustado da TIS; $\lambda_{c}$ : peso fatorial dos itens do modelo completo da TIS; DP: desvio padrão.

* Valores discrepantes de assimetria e curtose. 
O modelo fatorial completo da TIS (43 itens) não apresentou bom ajustamento em nenhuma das amostras (Feminina: $\lambda=0,46-0,93, \chi^{2} / \mathrm{gl}=5,70, \mathrm{RMSEA}[\mathrm{IC} 90 \%]=0,10[0,09-0,10], \mathrm{CFI}=0,86, \mathrm{TLI}=0,86$; Masculina: $\lambda=0,44-0,92, \chi^{2} / \mathrm{gl}=2,97$, RMSEA[IC90\%] $=0,08[0,07-0,08]$, CFI = 0,87, TLI = 0,86). As correlações $(r)$ entre os fatores da TIS foram todas significativas $(\mathrm{p}<0,001)$ tanto para a amostra feminina ( $\mathrm{r}$ : pais $\mathrm{x}$ mídia $=0,28$, amigos $\mathrm{x}$ mídia $=0,33$, amigos $\mathrm{x}$ pais $=0,42$ ) quanto para a amostra masculina ( $\mathrm{r}$ : pais $\mathrm{x}$ mídia $=0,43$, amigos $\mathrm{x}$ mídia $=0,46$, amigos $\mathrm{x}$ pais $=0,46$ ). Para melhorar o ajustamento desse modelo, os índices de modificação foram analisados. Os itens 4, 27, 34 e 41 apresentaram fortes correlações com vários outros itens de fatores diferentes em ambas as amostras, indicando falta de especificidade, e, portanto, esses foram excluídos. Foram identificadas e inseridas quatro correlações entre os erros dos itens 1 e 2, 7 e 10, 19 e 20 e 42 e 43 em ambas as amostras, uma vez que todas eram de itens do mesmo fator. Após a realização dessas modificações, o modelo apresentou adequado ajustamento às amostras (Feminina: $\lambda=0,44-0,90, \chi^{2} / \mathrm{gl}=4,53$, RMSEA[IC90\%] $=0,08[0,08-0,09], \mathrm{CFI}=0,92$, $\mathrm{TLI}=0,91$; Masculina: $\lambda=0,58-0,87, \chi^{2} / \mathrm{gl}=2,73$, RMSEA[IC90\%] $=0,08[0,07-0,08]$, CFI $=0,90$, TLI = $0,90)$, e as correlações entre os fatores também foram significativas ( $<<0,001)$ tanto para as mulheres ( $\mathrm{r}$ : pais $\mathrm{x}$ mídia $=0,30$, amigos $\mathrm{x}$ mídia $=0,36$, amigos $\mathrm{x}$ pais $=0,43$ ) quanto para os homens ( $\mathrm{r}$ : pais $\mathrm{x}$ mídia $=0,45$, amigos $\mathrm{x}$ mídia $=0,47$, amigos $\mathrm{x}$ pais $=0,45)$. Os pesos fatoriais dos itens da TIS foram adequados em mulheres e homens (Tabela 2).

Após ajustamento do modelo aos dados, observou-se a validade convergente, e os valores de VEM estiveram adequados nos fatores mídia (Feminina e Masculina: VEM =0,56) e amigos (Feminina e Masculina: VEM = 0,59) e limítrofes no fator pais (Feminina: VEM = 0,48 e Masculina: VEM = 0,49). Quanto à validade discriminante $\left(\mathrm{r}^{2}=0,09-0,18\right)$ e a confiabilidade $(\alpha=0,88-0,94$; CC $=0,91-0,95)$, todos os valores foram adequados em todos os fatores da TIS em ambas as amostras.

Os escores médios da influência da mídia (Feminina: média $(\mathrm{M})=2,35[\mathrm{DP}=0,80]$; Masculina: $\mathrm{M}=1,80[\mathrm{DP}=0,64]$; $\mathrm{p}<0,001$ ), pais (Feminina: $\mathrm{M}=2,02[\mathrm{DP}=0,67]$; Masculina: $\mathrm{M}=1,91[\mathrm{DP}=0,62]$; $\mathrm{p}=0,035$ ) e amigos (Feminina: $\mathrm{M}=2,44[\mathrm{DP}=0,79]$; Masculina: $\mathrm{M}=2,10[\mathrm{DP}=0,72]$; $\mathrm{p}<0,001$ ) foram significativamente diferentes entre as amostras. As mulheres apresentaram maiores escores em todos os fatores.

$\mathrm{Na}$ Tabela 3, encontra-se a prevalência de estudantes classificados de acordo com o grau de influência da mídia, dos pais e dos amigos na aparência corporal dos estudantes de acordo com o sexo. Nota-se que a maioria dos estudantes de ambos os sexos foi classificada de muito baixa a baixa influência da mídia, pais e amigos na aparência corporal. Em relação à influência da mídia, nota-se que as prevalências nas categorias moderada e alta foram significativamente maiores entre as mulheres. Para o fator pais, não houve diferença nas prevalências entre os sexos. Ainda, as mulheres sofrem maior influência dos amigos do que os homens.

\section{Tabela 3}

Classificação dos estudantes universitários segundo os escores médios dos fatores da Tripartite Influence Scale (TIS) separados por sexo.

\begin{tabular}{|c|c|c|c|c|c|}
\hline \multirow[t]{2}{*}{ Fator } & \multirow[t]{2}{*}{ Classificação } & \multicolumn{2}{|c|}{ Feminino } & \multicolumn{2}{|c|}{ Masculino } \\
\hline & & n (\%) & IC95\% & n (\%) & IC95\% \\
\hline \multirow[t]{4}{*}{ Mídia } & Muito baixa & $199(40,4)$ & $36,4-44,9$ & $218(72,9)$ & $68,2-77,6$ \\
\hline & Baixa & $208(42,3)$ & $38,0-46,3$ & $65(21,7)$ & $17,1-26,8$ \\
\hline & Moderada & $65(13,2)$ & $10,4-16,3$ & $16(5,4)$ & $3,0-8,0$ \\
\hline & Alta & $20(4,1)$ & $2,4-5,9$ & - & - \\
\hline \multirow[t]{4}{*}{ Pais } & Muito baixa & $285(57,9)$ & $53,5-62,2$ & $189(63,2)$ & $57,5-68,9$ \\
\hline & Baixa & $163(33,1)$ & $29,1-37,2$ & $93(31,1)$ & $26,1-36,5$ \\
\hline & Moderada & $41(8,3)$ & $6,1-10,8$ & $15(5,0)$ & $2,7-7,4$ \\
\hline & Alta & $3(0,6)$ & $0,0-1,4$ & $2(0,7)$ & $0,0-1,7$ \\
\hline \multirow[t]{4}{*}{ Amigos } & Muito baixa & $183(37,2)$ & $32,9-41,7$ & $157(52,5)$ & $46,8-57,9$ \\
\hline & Baixa & $198(40,2)$ & $36,2-45,1$ & $108(36,1)$ & $31,1-41,5$ \\
\hline & Moderada & $92(18,7)$ & $15,4-22,4$ & $31(10,4)$ & $7,0-13,7$ \\
\hline & Alta & $19(3,9)$ & $2,2-5,7$ & $3(1,0)$ & $0,0-2,3$ \\
\hline
\end{tabular}

IC95\%: intervalo de 95\% de confiança. 
A Tabela 4 apresenta as estimativas obtidas no modelo de regressão para verificar a relação das variáveis demográficas e antropométricas com os fatores da TIS. Para a amostra feminina, foi identificado que a atividade laboral, o consumo de suplementos alimentares para alterar o corpo, o IMC e o nível de atividade física foram significativas. As mulheres que não trabalham são mais influenciadas pela mídia, aquelas que consomem mais suplementos alimentares para alterar o corpo e apresentaram maior IMC foram mais influenciadas pelos pais. As estudantes com maior nível de atividade física sofreram maior influência dos amigos. Para a amostra masculina, foi identificado que a idade, o consumo de suplementos alimentares para alterar o corpo, o IMC e a autoavaliação da alimentação foram significativas. Observou-se que, quanto menor a idade e maior o consumo de suplementos alimentares, maior foi a influência da mídia. Ainda, quanto maior o IMC, maior a influência dos pais na aparência corporal dos estudantes, e aqueles que classificaram melhor sua alimentação foram os mais influenciados pelos amigos.

\section{Discussão}

Este estudo apresentou, pela primeira vez, os indicadores psicométricos da TIS obtidos por meio de estratégia confirmatória em estudantes universitários. A TIS está disponível para uso em estudos nacionais/internacionais, mas pouco se sabia, até o momento, sobre sua validade fatorial. Além disso, foi proposta estratégia para definição de pontos de corte para cada fator da escala, e foram identificadas características amostrais que influenciam nesses fatores e devem ser consideradas frente à avaliação clínica e/ou epidemiológica dos aspectos interferentes na aparência corporal de jovens universitários. Assim, espera-se que esse estudo possa auxiliar pesquisadores e clínicos para uma tomada de decisão mais direcionada quando da avaliação de aspectos socioculturais relacionados à aparência corporal.

Antes da aplicação do instrumento, o processo de validação de face da TIS foi realizado, e, nessa etapa, modificações nas opções de resposta da escala foram realizadas visando contribuir para a captura do conceito. A escala com as respostas adaptadas foi pré-testada e considerada adequada. Assim, quando da aplicação da TIS, sugere-se a utilização das opções de resposta e das codificações descritas no tópico de validação de face deste estudo.

Em relação ao ajustamento da TIS aos dados, nós utilizamos análise fatorial confirmatória, uma vez que o modelo teórico já estava previamente estabelecido. Nós verificamos que o modelo original não se ajustou aos dados e foi modificado visando garantir a qualidade da medida avaliada. Cabe esclarecer que a realização de modificações para ajustamento de modelos é comum, pois a validade não é uma propriedade do instrumento por si, mas sim dos dados recolhidos 29,34. Após algumas modificações na TIS, observou-se adequado ajustamento tanto para a amostra feminina quanto para a masculina, apontando validade do modelo para os dados. Ressalta-se ainda que, do ponto de vista configuracional, o modelo ajustado foi idêntico para ambos os sexos. Os estudos que utilizam a TIS não avaliaram o ajustamento do modelo original às amostras e, portanto, não foi possível comparar nossos indicadores psicométricos com a literatura. Com relação à confiabilidade, destaca-se que o modelo ajustado apresentou bons indicadores, o que corrobora com estudos anteriores 6,18,21. Contudo, alerta-se que a confiabilidade só deve ser atestada após avaliadas e comprovadas as estimativas das validades do instrumento na amostra 22. Assim, sugere-se que os pesquisadores/clínicos avaliem cuidadosamente os estudos científicos da TIS e se atentem à necessidade de verificação dos indicadores psicométricos do instrumento antes de sua utilização em diferentes amostras ou contextos.

Após definir o modelo de medida com adequado ajustamento aos dados, foram propostos pontos de corte considerando os percentis da escala de resposta da TIS. Essa estratégia foi delineada para disponibilizar faixas que possibilitem agrupar os indivíduos segundo o grau de influência da mídia, dos pais e dos amigos na aparência corporal e, assim, facilitar a interpretação e comparação dos dados obtidos em âmbito clínico e epidemiológico. Em relação aos escores médios, nós observamos diferenças significativas entre os sexos, sendo que as mulheres apresentaram maiores valores, ou seja, foram mais influenciadas pela mídia, pais e amigos. Esse resultado corrobora com estudos anteriores 39,40,41,42 revelando que existem diferenças expressivas entre mulheres e homens quanto à influência 
Tabela 4

Modelo de regressão múltiplo multivariado testado em estudantes universitários para cada sexo.

\begin{tabular}{|c|c|c|c|c|c|c|}
\hline \multirow[t]{2}{*}{ Variável independente (demográficas) $\rightarrow$ Variável dependente (fatores da TIS) } & \multicolumn{3}{|c|}{ Completo } & \multicolumn{3}{|c|}{ Refinado } \\
\hline & $\beta$ & EP & Valor de $p$ & $\beta$ & EP & Valor de $\mathrm{p}$ \\
\hline \multicolumn{7}{|l|}{ Feminina } \\
\hline Idade $\rightarrow$ Mídia & $-0,114$ & 0,042 & 0,006 * & - & - & - \\
\hline Idade $\rightarrow$ Pais & 0,074 & 0,052 & 0,151 & - & - & - \\
\hline Idade $\rightarrow$ Amigos & $-0,025$ & 0,054 & 0,647 & - & - & - \\
\hline Atividade laboral $\rightarrow$ Mídia & $-0,080$ & 0,041 & 0,050 * & $-0,185$ & 0,048 & $<0,001$ * \\
\hline Atividade laboral $\rightarrow$ Pais & 0,032 & 0,049 & 0,521 & - & - & - \\
\hline Atividade laboral $\rightarrow$ Amigos & $-0,031$ & 0,052 & 0,541 & - & - & - \\
\hline Consumo de suplementos alimentares $\rightarrow$ Mídia & 0,626 & 0,021 & $<0,001$ * & - & - & - \\
\hline Consumo de suplementos alimentares $\rightarrow$ Pais & 0,261 & 0,070 & $<0,001 *$ & 0,173 & 0,047 & $<0,001$ * \\
\hline Consumo de suplementos alimentares $\rightarrow$ Amigos & $-0,030$ & 0,080 & 0,705 & - & - & - \\
\hline Autoavaliação de alimentação $\rightarrow$ Mídia & $-0,020$ & 0,041 & 0,627 & - & - & - \\
\hline Autoavaliação de alimentação $\rightarrow$ Pais & $-0,009$ & 0,050 & 0,865 & - & - & - \\
\hline Autoavaliação de alimentação $\rightarrow$ Amigos & 0,011 & 0,053 & 0,833 & - & - & - \\
\hline Índice de massa corporal $\rightarrow$ Mídia & 0,058 & 0,041 & 0,160 & - & - & - \\
\hline Índice de massa corporal $\rightarrow$ Pais & 0,111 & 0,050 & 0,026 * & 0,107 & 0,047 & 0,024 * \\
\hline Índice de massa corporal $\rightarrow$ Amigos & $-0,047$ & 0,052 & 0,361 & - & - & - \\
\hline Nível de atividade física $\rightarrow$ Mídia & $-0,023$ & 0,040 & 0,561 & - & - & - \\
\hline Nível de atividade física $\rightarrow$ Pais & 0,035 & 0,049 & 0,476 & - & - & - \\
\hline Nível de atividade física $\rightarrow$ Amigos & 0,100 & 0,050 & 0,048 * & 0,115 & 0,048 & 0,016 * \\
\hline Classe econômica $\rightarrow$ Mídia & $-0,115$ & 0,040 & 0,004 * & - & - & - \\
\hline Classe econômica $\rightarrow$ Pais & 0,011 & 0,049 & 0,822 & - & - & - \\
\hline Classe econômica $\rightarrow$ Amigos & 0,037 & 0,051 & 0,467 & - & - & - \\
\hline \multicolumn{7}{|l|}{ Masculino } \\
\hline Idade $\rightarrow$ Mídia & $-0,181$ & 0,066 & 0,006 * & $-0,151$ & 0,061 & 0,014 * \\
\hline Idade $\rightarrow$ Pais & 0,015 & 0,069 & 0,827 & - & - & - \\
\hline Idade $\rightarrow$ Amigos & $-0,083$ & 0,068 & 0,219 & - & - & - \\
\hline Atividade laboral $\rightarrow$ Mídia & 0,067 & 0,067 & 0,316 & - & - & - \\
\hline Atividade laboral $\rightarrow$ Pais & 0,001 & 0,068 & 0,985 & - & - & - \\
\hline Atividade laboral $\rightarrow$ Amigos & 0,057 & 0,068 & 0,404 & - & - & - \\
\hline Consumo de suplementos alimentares $\rightarrow$ Mídia & 0,135 & 0,063 & 0,032 * & 0,152 & 0,062 & 0,013 * \\
\hline Consumo de suplementos alimentares $\rightarrow$ Pais & 0,105 & 0,065 & 0,104 & - & - & - \\
\hline Consumo de suplementos alimentares $\rightarrow$ Amigos & 0,062 & 0,064 & 0,337 & - & - & - \\
\hline Autoavaliação da alimentação $\rightarrow$ Mídia & 0,107 & 0,064 & 0,093 & - & - & - \\
\hline Autoavaliação da alimentação $\rightarrow$ Pais & $-0,022$ & 0,065 & 0,738 & - & - & - \\
\hline Autoavaliação da alimentação $\rightarrow$ Amigos & 0,152 & 0,064 & 0,018 * & 0,165 & 0,061 & 0,007 * \\
\hline Índice de massa corporal $\rightarrow$ Mídia & 0,044 & 0,062 & 0,480 & - & - & - \\
\hline Índice de massa corporal $\rightarrow$ Pais & 0,128 & 0,063 & 0,043 * & 0,146 & 0,062 & 0,018 * \\
\hline Índice de massa corporal $\rightarrow$ Amigos & 0,078 & 0,063 & 0,214 & - & - & - \\
\hline Nível de atividade física $\rightarrow$ Mídia & $-0,080$ & 0,062 & 0,198 & - & - & - \\
\hline Nível de atividade física $\rightarrow$ Pais & $-0,022$ & 0,063 & 0,730 & - & - & - \\
\hline Nível de atividade física $\rightarrow$ Amigos & $-0,006$ & 0,063 & 0,919 & - & - & - \\
\hline Classe econômica $\rightarrow$ Mídia & 0,004 & 0,064 & 0,949 & - & - & - \\
\hline Classe econômica $\rightarrow$ Pais & 0,060 & 0,065 & 0,354 & - & - & - \\
\hline Classe econômica $\rightarrow$ Amigos & 0,062 & 0,064 & 0,957 & - & - & - \\
\hline
\end{tabular}

ß: estimativa padronizada; EP: erro padrão; TIS: Escala de Influência dos Três Fatores.

Nota: o modelo completo incluiu todas as variáveis demográficas, e o modelo refinado incluiu apenas as variáveis significativas.

$* p<0,05$ 
dos aspectos socioculturais na aparência corporal, o que reforça a necessidade de ações direcionadas para cada sexo.

Com relação aos resultados observados nas prevalências, nós identificamos que a maioria dos participantes apresentou de muito baixa a baixa influência da mídia, pais e amigos na aparência corporal, e, considerando que se trata de uma amostra não clínica, esse resultado era esperado. Contudo, vale destacar que, quando observadas as categorias alta e moderada entre os fatores, as estimativas chegam até 18,7\% de acometimento, e tal resultado alerta para a necessidade de ações de conscientização e intervenção para evitar instalação de problemas futuros quanto aos transtornos alimentares e de imagem corporal. Ainda, analisando as prevalências, foram observados, entre os estudantes, maiores valores para o grau moderado e alto entre as mulheres, tanto no que se refere à influência da mídia quanto à dos amigos. Esse resultado vai ao encontro da literatura em que é apontado que a maneira como são sentidas as pressões socioculturais e o padrão ideal de aparência é diferente para homens e mulheres 16,43. A investigação da influência da família, mídia e amigos na percepção corporal e alimentação dos indivíduos tem sido comumente apresentada na literatura, contudo, não foram encontrados, até o momento, estudos que utilizaram a TIS para essa comparação. Entretanto, as evidências relacionadas às pressões socioculturais em relação à aparência vão na mesma direção dos resultados do presente estudo, reforçando a importância dessas na vida das pessoas 39,44,45,46.

No que se refere à relação das características amostrais com os fatores avaliados pela TIS, destacase que as mulheres que não trabalham e os homens mais jovens e que consomem mais suplementos para alterar o corpo são mais influenciados pela mídia em relação à aparência corporal. Mellor et al. 41 apontam a relevância da mídia sobre as atitudes das pessoas diante da percepção corporal e destacam a preocupação e a necessidade de conscientização quanto às mensagens que são divulgadas nos meios de comunicação. Esse resultado também é sustentado por estudos mais recentes que enfatizam a forte influência da mídia social sobre a percepção corporal das pessoas 47,48.

A relação significativa entre a influência dos pais e o consumo de suplementos alimentares e o IMC também foi encontrada no presente estudo. Mellor et al. 42 relatam que os indivíduos com maior IMC são fortemente pressionados pela família para mudar o corpo, reforçando a necessidade de ações educativas voltadas aos membros da família para buscarem utilizar estratégias que possam minimizar os efeitos de pressões estabelecidas de forma negativa. Já quanto ao consumo de suplementos alimentares e a influência dos pais, essa relação não é bem estabelecida na literatura, talvez seja uma estratégia adotada pelos indivíduos diante das mensagens impositivas recebidas de inadequação, contudo, mais estudos devem ser realizados para sustentar essa especulação. Ainda, verificamos a relação significativa entre maior nível de atividade física e melhor percepção da própria alimentação com a influência dos amigos na aparência corporal. Estudos anteriores relatam que o convívio social com amigos pode influenciar nas atitudes corporais 49 e alimentares 50 dos indivíduos. No presente estudo, a influência dos amigos representou um papel importante na aquisição de hábitos saudáveis dos estudantes. Assim, é interessante elaborar estratégias para incentivar formas inclusivas de diálogos sobre temas voltados à imagem corporal e à alimentação entre grupos de amigos. A partir da relevância observada das características amostrais nos fatores psicossociais relacionados à aparência corporal, destaca-se a importância de incorporação dessas características em protocolos direcionados às ações educativas, preventivas e de intervenção voltados à promoção da saúde da população.

Este estudo apresenta algumas limitações que devem ser mencionadas. A primeira refere-se à seleção da amostra que não foi probabilística nem representativa da população universitária do Brasil, limitando, portanto, a generalização dos resultados. A segunda refere-se ao desenho de estudo transversal que não permite estabelecer relações diretas de causa e efeito. Contudo, esse delineamento tem sido comumente utilizado em rastreamentos epidemiológicos. Para tentar minimizar essas limitações, foi utilizada uma amostra de dimensão superior ao mínimo estimado para realização das análises estatísticas, mas sugere-se a realização de outros estudos com amostras mais alargadas e/ou representativas da população brasileira. Ainda, uma terceira limitação deve ser mencionada sobre a utilização da TIS em contexto epidemiológico que pode ser entendida como difícil devido à quantidade de itens da escala (modelo original = 43 itens e modelo ajustado $=39$ itens). Assim, nós sugerimos a realização de estudos futuros com a TIS para investigar a possibilidade de desenvolver um modelo reduzido para a escala visando facilitar sua utilização em contexto epidemiológico. Apesar dessas limitações, esse foi o primeiro estudo que apresentou evidências relacionadas aos indicadores psicométricos da TIS 
aplicada a jovens universitários podendo ser, portanto, útil principalmente no sentido de despertar, entre os pesquisadores e clínicos da área, a necessidade de se verificar o ajustamento dos modelos teóricos dos instrumentos psicométricos para cada amostra/contexto e apresentar a validade dos dados em conjunto com suas análises.

\section{Conclusão}

A realização deste estudo permitiu a apresentação de evidências relacionadas à validade de construto e à confiabilidade da TIS em estudantes universitários brasileiros. Também foi apresentada uma proposta para cálculo das prevalências a partir do grau de influência da mídia, dos pais e dos amigos na aparência corporal que poderá ser utilizada em estudos futuros. Essa proposta poderá facilitar a avaliação das influências mencionadas em contexto clínico bem como a comparação de estimativas obtidas em estudos epidemiológicos. Além disso, foram identificadas diferentes variáveis que impactaram significativamente nos fatores da TIS e podem ser relevantes para a elaboração de protocolos clínicos ou de investigação mais direcionados. Ainda, vale mencionar, que, de um modo geral, as mulheres foram mais influenciadas pela mídia e pelos amigos do que os homens.

\section{Colaboradores}

W. R. Silva participou do desenvolvimento do projeto de pesquisa e desenho de estudo, coleta e análise dados e escrita do manuscrito enviado para publicação. J. Marôco e J. A. D. B. Campos participaram do desenvolvimento do projeto de pesquisa, desenho de estudo e revisão do manuscrito enviado para publicação.

\section{Informações adicionais}

ORCID: Wanderson Roberto da Silva (0000-00018897-8772); João Marôco (0000-0001-9214-5378); Juliana Alvares Duarte Bonini Campos (0000-00017123-5585).

\section{Agradecimentos}

À Faculdade de Ciências Farmacêuticas da Universidade Estadual Paulista (UNESP, campus de Araraquara) pelo apoio institucional (SisPROPe no 2082) e às estudantes Fernanda Cristina Maurício e Bianca Gonzalez Martins pelo auxílio na coleta e no preenchimento dos dados.

\section{Referências}

1. Cash TF, Smolak L. Body image: a handbook of science, practice, and prevention. 2nd Ed. New York: Guilford Press; 2011.

2. Martins DF, Nunes MFO, Noronha APP. Satisfação com a imagem corporal e autoconceito em adolescentes. Psicol Teor Pesqui 2008; 10:94-105.

3. van den Berg P, Thompson JK, ObremskiBrandon $\mathrm{K}$, Coovert $\mathrm{M}$. The tripartite influence model of body image and eating disturbance: a covariance structure modeling investigation testing the mediational role of appearance comparison. J Psychosom Res 2002; 53:1007-20

4. Thompson JK, Coovert MD, Stormer S. Body image, social comparison, and eating disturbance: a covariance structure modeling investigation. Int J Eat Disord 1999; 26:43-51.

5. Thompson JK, Heinberg LJ, Altabe MN, Tantleff-Dunn S. Exacting beauty: theory, assessment, and treatment of body image disturbance. Washington DC: American Psychological Association; 1999.

6. de Carvalho PHB, Alvarenga MDS, Ferreira MEC. An etiological model of disordered eating behaviors among Brazilian women. Appetite 2017; 116:164-72.

7. Tylka TL. Refinement of the tripartite influence model for men: dual body image pathways to body change behaviors. Body Image 2011; 8:199-207.

8. Silva WR, Dias JCR, Pimenta F, Campos JADB. Avaliação da preocupação com a forma corporal: um desafio. Psychology, Community \& Health 2014; 3:103-19. 
9. Cash TF, Morrow JA, Hrabosky JI, Perry AA. How has body image changed? A cross-sectional investigation of college women and men from 1983 to 2001. J Consult Clin Psychol 2004; 72:1081-9.

10. Lantz EL, Gaspar ME, DiTore R, Piers AD, Schaumberg K. Conceptualizing body dissatisfaction in eating disorders within a self-discrepancy framework: a review of evidence. Eat Weight Disord 2018; 23:275-91.

11. Laporta-Herrero I, Jauregui-Lobera I, BarajasIglesias B, Santed-German MA. Body dissatisfaction in adolescents with eating disorders. Eat Weight Disord 2018; 23:339-47.

12. Caran LG, Santana DD, Monteiro LS, da Veiga GV. Disordered eating behaviors and energy and nutrient intake in a regional sample of Brazilian adolescents from public schools. Eat Weight Disord 2018; 23:825-32.

13. Amaral ACS, Ferreira MEC. Body dissatisfaction and associated factors among Brazilian adolescents: a longitudinal study. Body Image 2017; 22:32-8.

14. Stice E, Ziemba C, Margolis J, Flick P. The dual pathway model differentiates bulimics, subclinical bulimics, and controls: testing the continuity hypothesis. Behav Ther 1996; 27:53149.

15. Levine MP, Smolak L, Hayden H. The relation of sociocultural factors to eating attitudes and behaviors among middle school girls. J Early Adolesc 1994; 14:471-90.

16. Schaefer LM, Burke NL, Thompson JK, Dedrick RF, Heinberg LJ, Calogero RM, et al. Development and validation of the Sociocultural Attitudes Towards Appearance Questionnaire-4 (SATAQ-4). Psychol Assess 2015; 27:54-67.

17. Keery H, van den Berg P, Thompson JK. An evaluation of the tripartite influence model of body dissatisfaction and eating disturbance with adolescent girls. Body Image 2004; 1:23751.

18. Conti MA, Scagliusi F, Queiroz GKO, Hearst N, Cordás TA. Adaptação transcultural: tradução e validação de conteúdo para o idioma português do modelo da Tripartite Influence Scale de insatisfação corporal. Cad Saúde Pública 2010; 26:503-13.

19. Shroff H, Thompson JK. The tripartite influence model of body image and eating disturbance: a replication with adolescent girls. Body Image 2006; 3:17-23.

20. Urbán R, Czeglédi E, Babusa B, Túry F. Testing the tripartite influence model of body image and eating disturbance among Hungarian adolescents. Body Image 2013; 10:232-42.

21. Amaral ACS, Ferreira MEC, Scagliusi FB, Costa LS, Cordas TA, Conti MA. Avaliação psicométrica da Escala de Influência dos Três Fatores (EITF). Psicol Reflex Crit 2013; 26:213-24.

22. Campos JADB, Marôco J. Modelagem de equações estruturais: aplicação à validação de instrumentos psicométricos. In: Damásio BF, Borsa JC, organizadores. Manual de desenvolvimento de instrumentos psicológicos. São Paulo: Vetor; 2017. p. 323-45.
23. Kakeshita IS, Almeida SS. Relação entre índice de massa corporal e a percepção da autoimagem em universitários. Rev Saúde Pública 2006; 40:497-504.

24. Laus MF, Costa TMB, Almeida SS. Distorção da imagem corporal em adolescentes: um estudo de comparação entre dois instrumentos. Medicina 2009; 42:358-65.

25. Legnani RFS, Legnani E, Pereira EF, Gasparotto GS, Vieira LF, Campos W. Transtornos alimentares e imagem corporal em acadêmicos de Educação Física. Motricidade 2012; 18:84-91.

26. Miranda VPN, Filgueiras JF, Neves CM, Teixeira PC, Ferreira MEC. Insatisfação corporal em universitários de diferentes áreas de conhecimento. Rev Bras Psiquiatr 2012; 61:25-32.

27. Silva WR, Dias JCR, Maroco J, Campos JADB. Factors that contribute to the body image concern of female college students. Rev Bras Epidemiol 2015; 18:785-97.

28. Da Silva WR, Campos JADB, Marôco J. Impact of inherent aspects of body image, eating behavior and perceived health competence on quality of life of university students. PLoS One 2018; 13:e0199480.

29. Hair Jr. JF, Black WC, Babin B, Anderson RE. Multivariate data analysis. 7th Ed. Upper Saddle River: Prentice Hall; 2009.

30. De Onis M, Onyango AW, Borghi E, Siyam A, Nishida C, Siekmann J. Development of a WHO growth reference for school-aged children and adolescents. Bull World Health Organ 2007; 85:660-7.

31. World Health Organization. Obesity: preventing and managing the global epidemic. Geneva: World Health Organization; 2000. (WHO Technical Report Series, 894).

32. Reichenheim ME, Moraes CL. Operationalizing the cross-cultural adaptation of epidemological measurement instruments. Rev Saúde Pública 2007; 41:665-73.

33. Beaton DE, Bombardier C, Guillemin F, Ferraz MB. Guidelines for the process of cross-cultural adaptation of self-report measures. Spine 2000; 25:3186-91.

34. Marôco J. Análise de equações estruturais. Pêro Pinheiro: ReportNumber; 2014.

35. Byrne BM. Structural equation modeling with Amos: basic concepts, applications and programming. New Jersey: Lawrence Erlbaum Associates; 2001.

36. Fornell C, Larcker DF. Evaluating structural equation models with unobservable variables and measurement error. J Mark Res 1981; 18:39-50.

37. Jorgensen TD, Pornprasertmanit S, Schoemann AM, Rosseel Y. semTools: useful tools for structural equation modeling. https:// CRAN.R-project.org/package $=$ semTools (acessado em 09/Jul/2018).

38. Rossel Y. lavaan: an $\mathrm{R}$ package for structural equation modeling. J Stat Softw 2012; 48:1-36.

39. McCabe MP, Ricciardelli LA. Sociocultural influences on body image and body changes among adolescent boys and girls. J Soc Psychol 2003; 143:5-26. 
40. McCabe MP, Ricciardelli LA, Finemore J. The role of puberty, media and popularity with peers on strategies to increase weight, decrease weight and increase muscle tone among adolescent boys and girls. J Psychosom Res 2002; 52:145-53.

41. Mellor D, McCabe M, Ricciardelli L, Merino ME. Body dissatisfaction and body change behaviors in Chile: the role of sociocultural factors. Body Image 2008; 5:205-15.

42. Mellor D, McCabe M, Ricciardelli L, Yeow J, Daliza N, Hapidzal NF. Sociocultural influences on body dissatisfaction and body change behaviors among Malaysian adolescents. Body Image 2009; 6:121-8.

43. Schaefer LM, Burke NL, Thompson JK. Thinideal internalization: how much is too much? Eat Weight Disord 2018; [Epub ahead of print].

44. Granero-Gallegosa A, Lucas JM, Sicilia A, Medina-Casaubónc J, Alcaraz-Ibáñeza M. Analysis of sociocultural stereotypes towards thin body and muscular body: differences according to gender and weight discrepancy. Rev Psicodidáct 2018; 23:26-32.

45. McCabe MP, Ricciardelli LA, Holt K. Are there different sociocultural influences on body image and body change strategies for overweight adolescent boys and girls? Eat Behav 2010; 11:156-63.
46. Xu X, Mellor D, Kiehne M, Ricciardelli LA, McCabe MP, Xu Y. Body dissatisfaction, engagement in body change behaviors and sociocultural influences on body image among Chinese adolescents. Body Image 2010; 7:156-64.

47. Bell BT, Cassarly JA, Dunbar L. Selfie-objectification: self-objectification and positive feedback ("likes") are associated with frequency of posting sexually objectifying self-images on social media. Body Image 2018; 10:83-9.

48. Saunders JF, Eaton AA. Snaps, selfies, and shares: how three popular social media platforms contribute to the sociocultural model of disordered eating among young women. Cyberpsychol Behav Soc Netw 2018; 21:343-54.

49. Dunton GF, Liao E, Intille S, Huh J, Leventhal A. Momentary assessment of contextual influences on affective response during physical activity. Health Psychol 2015; 34:1145-53.

50. Thompson KA, Kelly NR, Schvey NA, Brady $\mathrm{SM}$, Courville AB, Tanofsky-Kraff M, et al. Internalization of appearance ideals mediates the relationship between appearance-related pressures from peers and emotional eating among adolescent boys and girls. Eat Behav 2017; 24:66-73. 
Abstract

The Tripartite Influence Scale (TIS) is used to assess the influence of social and family factors on individuals' body image. However, little evidence has been presented on the validity of the TIS construct. The study aimed to estimate the psychometric indicators in TIS and identify the degree of influence of the media, parents, and peers on body image in university students, considering different characteristics. University students of both sexes participated. The psychometric indicators in TIS were assessed for each sex using confirmatory factor analysis. Mean scores were calculated for each TIS factor. Prevalence rates for degree of influence were presented. A multivariate regression model was built to verify the relationship between target characteristics and TIS factor scores. The sample included 791 university students (63.2\% women) 18 to 40 years of age. TIS did not initially display a good fit to the samples and was therefore refined. After refinement, the scale showed adequate validity and reliability for women and men. Significant differences were observed in the mean scores between women and men, and in the prevalence rates the majority of the students were in the low category. Work, consumption of food supplements for body changes, age, body mass index, self-rated diet, and level of physical activity were significantly related to TIS factors. Women were generally more influenced than men by the media and peers. Characteristics identified as related to TIS can be relevant for inclusion in clinical and research protocols.

Body Image; Students; Validation Studies

\section{Resumen}

La Escala de Influencia de los Tres Factores (TIS) se utiliza para evaluar la influencia de aspectos sociales y familiares en la apariencia corporal de los individuos. A pesar de ello, en el constructo de la TIS se presentan pocos indicios de validez. El objetivo de este estudio fue estimar los indicadores psicométricos de la TIS e identificar el grado de influencia de los medios de comunicación, padres y amigos, en la apariencia corporal de universitarios, considerando diferentes características. Participaron universitarios de ambos sexos. Los indicadores psicométricos de la TIS se evaluaron para cada sexo, utilizando un análisis factorial confirmatorio. Se calcularon las puntuaciones medias de cada factor de la TIS. Se presentaron las prevalencias del grado de influencia. Se construyó un modelo de regresión múltiple multivariado para verificar la relación de características de interés con puntuaciones de los factores de la TIS. Participaron 791 universitarios $(63,2 \%$ mujeres $)$ de 18 a 40 años. La TIS no presentó un buen ajuste a las muestras y fue depurada. Tras la depuración se observó una validez adecuada y confiabilidad para mujeres y hombres. Se verificaron diferencias significativas entre las puntuaciones medias de mujeres y hombres, asimismo, en las prevalencias la mayoría de los estudiantes estuvo en la categoría baja. La actividad laboral, consumo de suplementos alimenticios para alterar el cuerpo, edad, indice de masa corporal, autoevaluación de la alimentación y nivel de actividad física se relacionaron significativamente con los factores de la TIS. En general, las mujeres estuvieron más influenciadas por los medios y por los amigos que los hombres. Las características de las muestras identificadas y relacionadas con los factores de la TIS pueden ser relevantes para su inclusión en protocolos clínicos $y$ de investigación.

Imagen Corporal; Estudantes; Estudios de Validación
Recebido em 05/Out/2018

Versão final reapresentada em 15/Dez/2018

Aprovado em 06/Fev/2019 